Method Fifty workers from two yacht factories participated, including 23 FRP laminators and 27 non-FRP workers. Pre-shift colour confusion index (CCI) was measured on the first workday of a week by using Lanthony Desaturated Panel D-15d. Concentrations of volatile organic compounds related to FRP laminating were collected by a stainless canister, and then analysed using a GC/MS. Cumulative working hours for FRP laminating was obtained from daily administrative records of the companies. Logistic regression was used to assess correlation between cumulative working hours (past $0.5,2$, and 8 years) of FRP laminating and pre-shift CCI, where work time and CCI were stratified by median and the model was adjusted for age and regular alcohol consumption.

Results The mean styrene exposure during FRP laminating was $4.2 \mathrm{ppm}$. The median of cumulative working hours for FRP laminating in the past $0.5,2$ and 8 years was $2,49,248 \mathrm{~h}$ respectively. Higher cumulative working hours for FRP laminating was associated with poor CCI (past 0.5 yrs, odd ratio $(\mathrm{OR})=3.1, \mathrm{p}$ $=0.1$; past 2 yrs, $\mathrm{OR}=4.8, \mathrm{p}=0.03$; past 8 yrs, $\mathrm{OR}=6.5, \mathrm{p}=$ $0.01)$. The effect of long-term exposure to styrene appeared to be stronger than short-term exposure.

Conclusions Long-term exposure to styrene from FRP laminating was associated with colour vision impairment.

\section{HEAD AND NECK CANCER AND OCCUPATIONAL EXPOSURE TO ASBESTOS, MINERAL WOOLS AND SILICA: RESULTS FROM THE ICARE STUDY}

${ }^{1}$ Sophie Paget-Bailly, 'Diane Cyr, 'Matthieu Carton, ${ }^{1}$ Florence Guida, ${ }^{1}$ Isabelle Stucker, ${ }^{2}$ Danièle Luce. ${ }^{1}$ INSERM U1018, Villejuif, France, ${ }^{2}$ INSERM U1085, Pointe À Pitre, France

\subsection{6/oemed-2014-102362.280}

Objectives To study the associations between head and neck cancer risk and occupational exposure to asbestos, mineral wools and silica.

Method ICARE is a population based case-control study conducted in France. Analyses were restricted to men and included 1833 cases of head and neck squamous cell carcinomas (HNSCC) and 2747 controls. Complete occupational history was collected. Occupational exposures were assessed through job-exposure matrices. Logistic models were used to estimate adjusted odds ratios (ORs) and 95\% confidence intervals (CI).

Results Exposure to asbestos was associated with an elevated risk of HNSCC $(\mathrm{OR}=1.9$, CI 1.6-2.3), and the risk increased significantly with the probability, duration and cumulative level of exposure. Significantly increased risks were found for all cancer sites: larynx $(\mathrm{OR}=2.1, \mathrm{CI} 1.6-2.8)$, hypopharynx $(\mathrm{OR}=2.0$ CI 1.5-2.8), oropharynx $(\mathrm{OR}=1.6 \mathrm{CI}=1.3-2.1)$ and oral cavity $(\mathrm{OR}=1.9$ CI 1.4-2.6). Conversely, after adjustment for asbestos exposure, exposure to mineral wools was not associated with HNSCC risk $(\mathrm{OR}=0.8 \mathrm{CI} 0.6-1.0)$, for any of the cancer sites. Exposure to silica was not associated with HNSCC risk overall $(\mathrm{OR}=0.9$ CI $0.7-1.2)$, but non-significantly elevated ORs were observed for the highest level of cumulative exposure for oropharyngeal $(\mathrm{OR}=1.6 \mathrm{CI} 0.8-3.2)$ and hypopharyngeal cancer $(\mathrm{OR}=1.9$ CI 0.9-4.1).

Conclusions Our findings confirm the role of asbestos exposure in laryngeal cancer, and suggest that asbestos exposure increases also the risk of oral and pharyngeal cancer. There is some evidence of an association between silica exposure and pharyngeal cancer. Exposure to mineral wools was not associated with HNSCC risk in our study.

\section{ESTIMATING THE PROPORTION OF OCCUPATIONAL CANCERS WITH MINIMAL RESOURCES: AN EXAMPLE FROM QUEBEC}

1,2 France Labrèche, ${ }^{1}$ Patrice Duguay, ${ }^{1}$ Alexandre Boucher, ${ }^{3}$ Robert Arcand. ${ }^{1}$ Institut de Recherche Robert-Sauvé en Santé Et en Sécurité Du Travail (IRSST), Montreal, Quebec, Canada; '2School of Public Health, Université de Montréal, Montreal, Quebec, Canada; ${ }^{3}$ Institut National de Santé Publique Du Québec, Montreal, Quebec, Canada

\subsection{6/oemed-2014-102362.281}

Objectives To estimate the number of work-related cancer cases and deaths in order to prioritise research activities.

Method Numbers of compensated incident cancers (between 2005-2007) and cancer deaths (between 1997-2005) were obtained from the Quebec Workers' Compensation Board. A second series of estimates was calculated by applying proportions of cancers attributable to work published for Finland (Nurminen and Karjalainen 2001) and for the United Kingdom (Rushton and colleagues 2012) to Quebec tumour registry data for 28 cancer sites. A comparison of industrial profiles of Finland, United Kingdom and Canada showed reasonable similarities between the countries over the last decades.

Results Compensation statistics reported an annual average of 94 incident cancers and 40 cancer deaths (98-99\% men), 60$64 \%$ of which being mesotheliomas, followed by respiratory cancers (30-37\%). Using published estimates of attributable fractions, it was estimated that $6.0 \%$ of incident cancers (men, 9.1\%; women, $2.7 \%$ ) and $7.6 \%$ of cancer deaths (men, 11.7\%; women, $2.8 \%$ ) could be attributable to work, resulting annually in 2200 new cancers and 1200 deaths. Incident cancers of the lungs, prostate, bladder, skin and breast (women) were the most numerous, whereas cancer sites resulting in more deaths were lung, breast (women) and mesothelium. On average, 53\% of incident mesothelioma cases were compensated yearly.

Conclusions This attempt at better estimating, albeit imperfectly, importance of the burden of cancer from occupational exposures can help prioritise research activities and increase stakeholders' awareness. However, better estimates of human impact and economic costs are warranted to justify large investments in preventive interventions.

\section{PSYCHOSOCIAL IMPACTS OF A CONTAMINATED NEEDLESTICK INJURY OR BLOOD AND BODY FLUID EXPOSURE IN HEALTH CARE WORKERS}

1,2Hsueh-Ching Wu, ${ }^{3}$ Weishan Chin, ${ }^{2}$ Judith Shu-Chu Shiao, ${ }^{3,4}$ Yue-Liang Leon Guo, ${ }^{5}$ Yueh-Tzu Hsieh. ${ }^{1}$ Department of Nursing, Hsin Sheng Junior College of Medical Care and Management, Taoyuan, Taiwan; 'Department of Nursing, College of Medicine, National Taiwan University (NTU) and NTU Hospital, Taipei, Taiwan; ${ }^{3}$ Institute of Occupational Medicine and Industrial Hygiene, College of Public Health, National Taiwan University, Taipei, Taiwan; ${ }^{4}$ Department of Environmental and Occupational Medicine, College of Medicine, National Taiwan University (NTU) and NTU Hospital, Taipei, Taiwan; ${ }^{5}$ Department of Nursing, Chung Hwa University of Medical Technology, Tainan, Taiwan

\subsection{6/oemed-2014-102362.282}

Objectives This study aims to explore the psychosocial impacts of health care workers (HCW) who were exposed to a contaminated needlestick Injury (NSI) or blood and body fluid (BBF) at work.

Method Personal interviews were performed using a phenomenological approach including in-depth interviews fort data collection, and expert meetings for data analysis. The 